\title{
Nonlinear Semi-Analytical Model for Simulation of Few-Mode Fiber Transmission
}

\author{
Filipe Ferreira, Student Member, IEEE, Sander Jansen, Senior Member, IEEE, \\ Paulo Monteiro, Member, IEEE, and Henrique Silva, Member, IEEE
}

\begin{abstract}
In this paper, a nonlinear semi-analytical model (NSAM) for simulation of few-mode fiber transmission is proposed. The NSAM considers the mode mixing arising from the Kerr effect and waveguide imperfections. An analytical explanation of the model is presented, as well as simulation results for the transmission over a two mode fiber (TMF) of $112 \mathrm{~Gb} / \mathrm{s}$ using coherently detected polarization multiplexed quadrature phase-shift-keying modulation. The simulations show that by transmitting over only one of the two modes on TMFs, long-haul transmission can be realized without increase of receiver complexity. For a $6000 \mathrm{~km}$ transmission link, a small modal dispersion penalty is observed in the linear domain, while a significant increase of the nonlinear threshold is observed due to the large core of TMF.
\end{abstract}

Index Terms - Nonlinear Fiber Optics, Optical Fibers, Optical Fiber Communication, Multimode Fibers, Few-Mode Fibers.

\section{INTRODUCTION}

$\mathrm{T}$ HE exponential increase in communications traffic is rapidly exhausting the optical fiber bandwidth available in the low loss window. In order to overcome this limit, considerable effort has been directed towards the development of advanced modulation formats, in order to enhance the spectral efficiency using denser Quadrature Amplitude Modulation (QAM) constellations [1,2]. However, modulation formats with denser constellations require a higher optical signal to noise ratio (OSNR) and have a reduced nonlinear tolerance. As a result, the feasible transmission distance of these modulation formats is significantly reduced. One radical solution is to increase the fiber core radius. However, as the fiber core radius increases the fiber becomes multimode.

Few-mode fibers (FMFs) have been proposed as a good

Manuscript received July 31, 2011. This work has been partially supported by: Nokia Siemens Networks S.A. Portugal, the Fundação para a Ciência e Tecnologia (FCT) under the grant SFRH/BDE/51094/2010, and the European Communities $7^{\text {th }}$ Framework Programme under grant agreement 228033 (MODE-GAP).

Filipe Ferreira and Paulo Monteiro are with Nokia Siemens Networks Portugal, S. A., Amadora and with Instituto de Telecomunicações, Portugal (email: filipe.ferreira@nsn.com and paulo.1.monteiro@nsn.com).

Sander Jansen is with the Nokia Siemens Networks GmbH \& Co. KG, Munich, Germany (email: sander.jansen@nsn.com).

Henrique Silva is with Instituto de Telecomunicações, Department of Electrical and Computer Engineering, University of Coimbra, Portugal (email: hjas@ co.it.pt). compromise between the decrease of the nonlinear coefficient and the introduced modal dispersion [3]. Due to the high mismatch of phase velocity between modes, these fibers present very weak mode coupling, such that long-haul single mode propagation has been already experimentally demonstrated [3]. Furthermore, FMFs have a theoretical larger information transport capacity, since each mode has a capacity which basically equals or doubles the capacity of a single mode fiber (SMF), depending of the mode degeneracy [4]. To take advantage of this additional capacity, Mode Division Multiplexing (MDM), has been proposed [5]. However, the complexity of the equalizer at the receiver is significantly higher than that for single mode transmission.

In this paper, we present a nonlinear semi-analytical model (NSAM) for simulation of FMF transmission, taking into account the mode coupling resulting from waveguide imperfections (Section II), and the Kerr nonlinear effects (Section III). Section IV provides the simulation setup and discussion of results. Conclusions are drawn in Section V.

\section{WAVEGUIDE IMPERFECTIONS}

The waveguide imperfections [6] caused by perturbations introduced during the fabrication process or by mechanical stresses imposed on the fiber in the field were modeled by random fluctuations of the core center position, given by: $\varepsilon_{r}(x, y, z)=\varepsilon_{r 0}(x+\delta x(z), y+\delta y(z), z)$, where $\varepsilon_{r}$ is the perturbed relative permittivity, $\varepsilon_{r 0}$ is the ideal relative permittivity, $\delta x$ and $\delta y$ are the random displacement of the abscissa and ordinate coordinates, respectively. The proposed model divides the fiber in multiple sections, each with a constant random displacement of the core center position. Therefore, each section has constant coupling coefficients. In order to analytically describe the crosstalk arising from the waveguide imperfections, we used the coupled wave theory [6], particularly the following coupled-mode equations:

$$
\begin{gathered}
\frac{\partial \tilde{A}_{\mu}}{\partial z}=-\mathrm{j}\left[\beta_{\mu}-\beta_{0 \mu}\right] \tilde{A}_{\mu}-\mathrm{j} \frac{\beta_{\mu}}{\left|\beta_{\mu}\right|} \sum_{v} C_{\mu \nu} \tilde{A}_{\nu} e^{\mathrm{j}\left(\beta_{0 \mu}-\beta_{0 v}\right) z} \\
C_{\mu \nu}=\frac{\omega \varepsilon_{0}}{4} \iint_{-\infty}^{+\infty}\left[\varepsilon_{r}(x, y)-\varepsilon_{r 0}(x, y)\right] \mathbf{E}_{\mu} \cdot \mathbf{E}_{v}^{*} d x d y
\end{gathered}
$$

where $\tilde{A}_{\mu}(z, \omega)$ is the Fourier transform of the mode $\mu$ slowly varying field envelope $A_{\mu}, \beta_{\mu}(\omega)$ is the mode $\mu$ propagation constant, and $\beta_{l \mu}$ is the $l^{\text {th }}$ order coefficient of a Taylor series 
expansion of $\beta_{\mu}(\omega)$ centered at the carrier frequency $\omega_{0}$. $C_{\mu \nu}$ are the coupling coefficients given by the area integral of the dot product of the electrical fields of mode $\mu$ and mode $v$, over the area where the permittivity difference between the ideal fiber $\left(\varepsilon_{r 0}\right)$ and the perturbed one $\left(\varepsilon_{r}\right)$ is nonzero.

Considering, the simple case of a TMF, where only the coupling between the $\mathrm{LP}_{01}$ mode $(\mu=1)$ and the $\mathrm{LP}_{11}$ mode $(\mu=2)$ is present, (1) can be written in a simpler form that can be solved analytically in each section [6]:

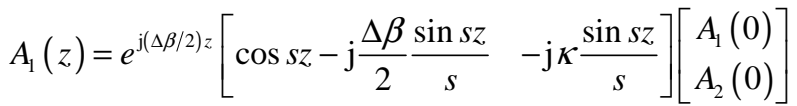

$$
\begin{aligned}
& A_{2}(z)=e^{-\mathrm{j}(\Delta \beta / 2) z}\left[-\mathrm{j} \kappa^{*} \frac{\sin s z}{s} \quad \cos s z+\mathrm{j} \frac{\Delta \beta}{2} \frac{\sin s z}{s}\right]\left[\begin{array}{l}
A_{1}(0) \\
A_{2}(0)
\end{array}\right]
\end{aligned}
$$

where $\Delta \beta=\beta_{1}-\beta_{2}, s^{2}=\kappa \kappa^{*}+(\Delta \beta / 2)^{2}$, and $\kappa=C_{12}=C_{21} *$. From (3) and (4), it can be concluded that the coupling strength depends on the relation between $|\kappa|^{2}$ and $\Delta \beta^{2}$.

A uniform distribution was assumed for the random position $\left(r_{d}, \theta_{d}\right)$ of the core center. In order to get a typical value for the maximum random displacement $\left(r_{d, \max }\right)$, the proposed model was applied to high birefringence ( $\mathrm{HiBi}$ ) fibers and the results were compared with published experimental measurements. The characteristics of the FlatCladd PMF fibers shown in table I of [7] were considered. Their birefringence is $B=\Delta n=2.5 \times 10^{-4}$, and the mode coupling parameter is $h=5.9$ $\mathrm{x} 10^{-6}$. The mode extinction ratio (MER) along the fiber length $l$ is given as a function of $h$ by $M E R=10 \log _{10}(\tanh (h l))$ [7], which is used to fit experimental results.

Finally, the simulation results and the experimental fitted function were compared. For a $r_{d, \max }$ of $1 \%$ of the core radius $\left(r_{\text {core }}\right)$ and a 50 meter simulation step size, considering perfect launch coupling to one of the modes, a very good agreement of the evolution of the power in each of the modes along the fiber length was obtained. Therefore, this model was adopted for the transmission simulations described in section IV.

\section{KERR NONLINEAR EFFECTS}

The Kerr effects were modeled by deriving the nonlinear pulse propagation equation for a multimode fiber, starting by writing the induced nonlinear polarization as a function of the electric field expansion into the two orthogonal polarization components of the $N$ orthogonal modes supported by the fiber. Following a derivation similar to the one present in [8] for single mode fibers, the generalized coupled nonlinear Schrödinger equations can then be written as:

$$
\begin{aligned}
& \frac{\partial A_{\mu i}}{\partial z}+\beta_{1 \mu i} \frac{\partial A_{\mu i}}{\partial t}-\frac{\mathrm{j} \beta_{2 \mu i}}{2} \frac{\partial A_{\mu i}}{\partial t}+\frac{\alpha_{\mu i}}{2} A_{\mu i}= \\
& -\mathrm{j}\left[\gamma_{\mu \mu i i}\left|A_{\mu i}\right|^{2}+2 \gamma_{\mu v i i} \sum_{\substack{v \neq \mu \\
v \neq \mu}}\left|A_{v i}\right|^{2}+\frac{2}{3} \gamma_{\mu v i j} \sum_{v}\left|A_{v j}\right|^{2}\right] A_{\mu i}
\end{aligned}
$$

where $i$ and $j$ are the orthogonal states of polarization of each mode $\mu$. $A_{\mu i}(z, t), \beta_{1 \mu i}, \beta_{2 \mu i}$ and $\alpha_{\mu i}$ are the slowly varying field envelope, group velocity parameter, group velocity dispersion parameter and attenuation parameter for the $i$ polarization of the $\mu$ mode, respectively. $\gamma_{\mu v i j}$ is the nonlinear coupling parameter between the $i$ polarization of mode $\mu$ and the $j$ polarization of mode $v$, which depends on the nonlinear refractive index $n_{2}$ of the silica, approximately $2.6 \times 10^{-26} \mathrm{~m}^{2} / \mathrm{W}$ [8], and on the intermodal effective area, and is given by:

$$
\gamma_{\mu v i j}=n_{2} \frac{\omega_{0}}{c} \frac{\iint_{-\infty}^{+\infty}\left|E_{\mu i}\right|^{2}\left|E_{v j}\right|^{2} d x d y}{\left(\iint_{-\infty}^{+\infty}\left|E_{\mu i}\right|^{2} d x d y\right)\left(\iint_{-\infty}^{+\infty}\left|E_{v j}\right|^{2} d x d y\right)}
$$

where $E_{\mu i}(x, y)$ is the mode field transverse distribution for the $i$ polarization of mode $\mu$.

In (5), the first term of the right side is responsible for modal self-phase modulation (mSPM) of the polarization $i$ of the mode $\mu$. The second term results in modal cross-phase modulation (mXPM) from the same polarization (i) of different waveguide modes $(\nu \neq \mu)$. The third term results also in $\mathrm{mXPM}$, but coming from the orthogonal polarization $(j)$ of the same $(\nu=\mu)$ or different waveguide modes $(\nu \neq \mu)$.

Finally, considering simultaneously the nonlinear contribution to the permittivity and the linear perturbation of the permittivity due to fiber structure imperfections, a novel integrated model was derived. Following the reasoning used in (1) and (5), the result obtained after some algebra was:

$$
\begin{aligned}
& \frac{\partial A_{\mu i}}{\partial z}+\beta_{1 \mu i} \frac{\partial A_{\mu i}}{\partial t}-\frac{\mathrm{j} \beta_{2 \mu i}}{2} \frac{\partial A_{\mu i}}{\partial t}+\frac{\alpha_{\mu i}}{2} A_{\mu i}= \\
& -\mathrm{j}\left[\gamma_{\mu \mu i i}\left|A_{\mu i}\right|^{2}+2 \gamma_{\mu v i i} \sum_{v \neq \mu}\left|A_{v i}\right|^{2}+\frac{2}{3} \gamma_{\mu v i j} \sum_{v}\left|A_{v j}\right|^{2}\right] A_{\mu i} \\
& -\mathrm{j} \frac{\beta_{\mu i}}{\left|\beta_{\mu i}\right|} \sum_{v a} C_{\mu v i a} A_{v a} e^{\mathrm{j}\left(\beta_{0 \mu i}-\beta_{0 v a}\right) z}
\end{aligned}
$$

In order to solve (5) and/or (7), in a nonlinear dispersive media, the split-step Fourier method was used.

\section{SIMULATION SETUP, RESULTS AND DISCUSSION}

In this section, the weak nonlinearity of a TMF was evaluated through the transmission of traffic over one of the supported modes, $\mathrm{LP}_{01}$ or $\mathrm{LP}_{11}$. The fiber considered has a step-index profile with a cladding refractive index of 1.46, a relative index gradient at the core-cladding interface $\Delta=\left(n_{\text {co }}\right.$ $\left.n_{\mathrm{cl}}\right) / n_{\mathrm{co}}=2.5 \times 10^{-3}$, and a core radius of $9 \mu \mathrm{m}$. By solving the Maxwell equations for these parameters, and using the weak guiding approximation, was obtained an effective mode index difference of $\Delta n \approx 1.32 \times 10^{-3}$, consequently a modal delay of $\sim 4 \mathrm{ps} / \mathrm{m}$. For this fiber the average MER obtained at the end of $75 \mathrm{~km}$. using (3) and (4), is $\sim-20 \mathrm{~dB}$, for $r_{d, \max }=0.01 \times r_{\text {core }}$. Table I gathers some of the TMF properties at $1550 \mathrm{~nm}$, together with those of the SMF used as reference for performance comparison.

The simulations were performed considering the LP 
approximate modes. The phase relation variations between the exact modes of the decomposition of $\mathrm{LP}_{11}\left(\mathrm{TM}_{01}, \mathrm{TE}_{01}\right.$ and $\mathrm{HE}_{21}$ ) [4], was accounted for during the simulations as polarization mode dispersion (PMD), as shown in Table I.

TABLE I

FIBER PROPERTIES AT $1550 \mathrm{NM}$.

\begin{tabular}{lllll}
\hline \hline Property & Unit & $\begin{array}{l}\text { TMF } \\
\mathbf{L P}_{\mathbf{0 1}}\end{array}$ & $\begin{array}{l}\text { TMF } \\
\mathbf{L P}_{\mathbf{1 1}}\end{array}$ & $\begin{array}{l}\text { SMF } \\
\mathbf{L P}_{\mathbf{0 1}}\end{array}$ \\
\hline Dispersion & $\mathrm{ps} /(\mathrm{nm} \mathrm{km})^{2}$ & 22.64 & 20.86 & 15.35 \\
Dispersion slope & $\mathrm{ps} /\left(\mathrm{nm}^{2} \mathrm{~km}\right)$ & 0.063 & 0.053 & 0.057 \\
$\boldsymbol{A}_{\text {eff }}$ & $\mu \mathrm{m}^{2}$ & 206.7 & 322.9 & 91.4 \\
$\boldsymbol{\gamma}$ - intramodal & $\mathrm{W}^{-1} / \mathrm{km}$ & 0.510 & 0.326 & 1.153 \\
$\boldsymbol{\gamma}$ - intermodal & $\mathrm{W}^{-1} / \mathrm{km}$ & 0.314 & 0.314 & - \\
PMD & $\mathrm{ps} / \sqrt{\mathrm{km}}$ & 0.069 & 0.687 & 0.069 \\
Attenuation & $\mathrm{dB} / \mathrm{km}$ & 0.22 & 0.22 & 0.22 \\
\hline \hline
\end{tabular}

In the transmitter/receiver in order to launch/detect selectively one of the TMF modes, an adiabatic taper can be used to launch/detect the $\mathrm{LP}_{01}$, and for the $\mathrm{LP}_{11}$ long-period fiber gratings (LPFG) can be used [5]. This setup introduces slight additional complexity, and during simulations their performance was considered ideal. In all transmission simulations performed, periodic amplification with $75 \mathrm{~km}$ spans was assumed to be realized with single stage erbiumdoped fiber amplifiers (EDFA), where only the wanted mode was amplified and the unused mode was removed. The modulation format used was $112 \mathrm{~Gb} / \mathrm{s}$ coherently detected polarization multiplexed (CP) quadrature phase-shift-keying (QPSK). A single channel, centered over the $1550 \mathrm{~nm}$ wavelength was transmitted. The receiver considered was the blind coherent digital receiver presented in [9], using twofold oversampling and frequency-domain equalization to compensate for the bulk of accumulated chromatic dispersion. After this, the signal is retimed, demultiplexed, and equalized in a butterfly 13 taps FIR filter. Finally, the phase is recovered from the constellation diagram. A $45 \mathrm{GHz}$ second-order optical Gaussian filter and a $28 \mathrm{GHz}$ fifth-order electrical Bessel filter were considered. The bit-error-ratio (BER) after transmission was measured using Monte Carlo simulation, considering EDFAs with a spontaneous emission factor of 2 and a gain adjusted to compensate for the total span losses.

Fig. 1 shows $\log _{10}(\mathrm{BER})$ after $6000 \mathrm{~km}$ as a function of the average input power, considering a 50 meter step size and $r_{d, \max }=0.01 \mathrm{x} r_{\text {core }}$. In the linear region, the TMF results show a power penalty of less than $1 \mathrm{~dB}$, compared with the SMF, due to the linear mode coupling between $\mathrm{LP}_{01}$ and $\mathrm{LP}_{11}$ of the TMF. This low penalty (in the absence of nonlinear effects) confirms the weak coupling strength given by (3) and (4), since $|k|$ ranges from 0 to $\sim 60$, and $\Delta \beta \approx 5000$, and already confirmed experimentally in [3]. However, for high launch powers where the optical performance is dictated by nonlinear effects TMF clearly outperforms SMF. Compared to the nonlinear threshold of SMF an increase with $\sim 4.5 \mathrm{~dB}$ and $\sim 6.5 \mathrm{~dB}$ of the maximum optical launched power for a $\mathrm{BER}=3.8 \times 10^{-3}$ is observed for $\mathrm{LP}_{01}$ mode and $\mathrm{LP}_{11}$ mode, respectively. These simulations improvements are related with the higher effective area of the TMF modes, compared to the
SMF, as shown in Table I. This results demonstrate that by transmitting over only one of the two modes on TMF, longhaul transmission can be realized providing a significant increase in nonlinear threshold compared to transmission on SMF without increase of receiver complexity (except for the necessary mode converters). These enhancements are in line with the experimental results presented in [3] for transmission over the $\mathrm{LP}_{01}$ of a similar TMF.

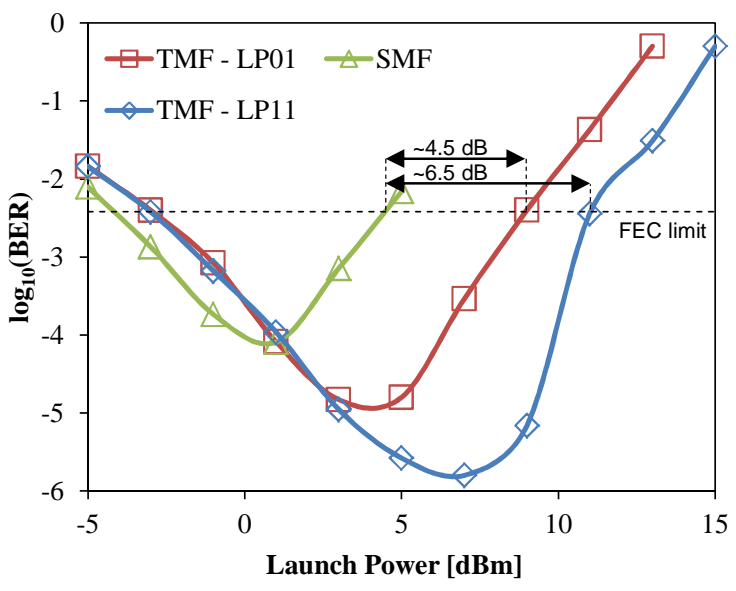

Fig. $1 . \log _{10}(\mathrm{BER})$ as function of the mode launch power.

\section{CONCLUSION}

This paper proposes a NASM for simulation of FMF transmission, including the modal coupling arising from waveguide imperfections and from the Kerr nonlinear effects. Therefore, this model is a valuable tool for the development of future high-capacity multimode fiber systems.

The simulation results obtained provide a validation of the weaker nonlinearity offered by TMFs. These fibers outperformed SMFs due to a much higher effective area, and a low modal dispersion penalty, showing that TMFs can be used for long-haul transmission without increase of receiver complexity (except for the necessary mode converters).

\section{REFERENCES}

[1] P. Winzer, and R. Essiambre, "Advanced Optical Modulation Formats," Proc. of the IEEE, vol. 94, no. 5, pp. 952-985, May 2006

[2] R. Essiambre, G. Kramer, P. Winzer, G. Foschini, and B. Goebel, "Capacity Limits of Optical Fiber Networks," J. Lightw. Technol., vol. 28, no. 4, pp. 662-701, Feb. 15, 2010

[3] F. Yaman, N. Bai, B. Zhu, T. Wang, and G. Li, "Long distance transmission in few-mode fibers," Optics Express, vol. 18, no. 12, pp. 13250-13257, 2010

[4] A. Ghatak, and K. Thyagarajan, An Introduction to Fiber Optics, Cambridge University Press, 1998, Chap. 8

[5] An Li, A. Al Amin, Xi Chen, and W. Shieh, "Reception of mode and polarization multiplexed 107-Gb/s CO-OFDM signal over a two-mode fiber," in $O F C$ 2011, San Diego, CA, PDPB8

[6] D. Marcuse, Theory of Dielectric Optical Waveguides, New York: Academic, 1974, Chap. 3 and 5

[7] J. Noda, K. Okamoto, and Y. Sasaki, "Polarization-maintaining fibers and their applications," J. Lightw. Technol., vol. 4, no. 8, pp. 1071-1089, Aug. 1986

[8] G. Agrawal, Nonlinear Fiber Optics, Academic Press, 2001, Chap. 8

[9] M. Kuschnerov, F. Hauske, K. Piyawanno, B. Spinnler, M. Alfiad, A. Napoli, and B. Lankl, "DSP for Coherent Single-Carrier Receivers," $J$. Lightw. Technol., vol. 27, no. 16, pp. 3614-3622, Aug. 15, 2009 